Gynäkol Geburtshilfliche Rundsch 1995;35:244

\title{
Sachregister Vol. 35, 1995
}

\section{Alternativmedizin 46}

Altes Allgemeines Krankenhaus Wien

153 Aminolävulinsäure $90 \delta$-Aminolävulinsäure 85 Archäologie 159 Arzt-Patientin-Beziehung 20 ATP-Tumorchemosensitivitätstest 85 Australien 42

Beckenbodeninsuffizienz 173 Beckenwandrezidiv 133 Blasenfunktionsstörung 221 Blutverlust 34 Bronzezeit 159 Brustkrebs, Geschichte 103

CD44-Splice-Varianten 16 Charité Berlin, Universitätskliniken

164 Chirurgiegeschichte $103 \mathrm{CC}^{1 / 8}$-Laserbehandlung 79

Diagnosestelhing 199

Druckanstieg, früher in der Harnröhre

als in der Blase 170 Dualdensitometrie 159 Ductus omphaloentericus 26 Dysplasien des

Genitales 90

Endogene Opioide 194 Endoskopische Technik 149 Endothelläsion 6 Extrauteringravidität 29

Fistelverschluss 226 Folsäure 2

Gebährhaus, Gebärhaus, Gebehrhaus

153 Genitale Papillomvirusinfektion 79 Geschichte der operativen Gynäkologie

164 Geschlechtspräferenz 20 Gestationsdiabetes 215 Glukose 215 Gynäkohistologie 188

Gynäkologische Onkologie 42, 139

Operationen 6,221

Untersuchung 20

Halsted, William Stewart 103

Hämostase 6

Hausgeburt 46

Heparin, niedermolekulares 6

Hippokrates von Kos 103

Homöopathie 46

Hormonsubstitution 72

Humanes $\beta$-Choriongonadotropin 38

Hypothalamische Amenorrhö 194

Hysterektomie 64, 142,226

Indikation 64, 142

Inguinalbestrahlung 209

Insulin 215

-, Bindung an Erythrozyten 12

Interferon-alfa-2a 79

Intravesikale Elektrostimulation 221

Jehovas Zeugen 34 Joseph II. 153

Knochendichte 159 Kolporrhaphia anterior 170 Kolposuspension nach Burch 175 Kortisol 194 Laparoskopie 139,226 Lymphknotentherapie 209 
Malignome 72 Mamma 98 Mammakarzinom 103 Mammatumor 98 Meckelsches Divertikel 26 Methotrexat 29

Nabelfistel 26 Nadelsuspension 170 Naloxon 194

Nebennierenrindenaktivität 194 Nervale Wucherungen 188 Neuralrohrdefekte 2

Operative Gynäkologie 149 - Therapie 175 Orale Kontrazeptiva 72, 199 Osteoporose 159

Östrogene 72

C-Peptid 215 Peyrilhe, Bernard 103 Photodynamische Lasertherapie 85, 90 Placental site trophoblastic tumor 38 Polyzystisches Ovarsyndrom 12 Prämenstruelle Beschwerden 199 Prämenstruelles Syndrom 199 Prävention 2 Prognoseparameter 16 Psychosexuelle Folgen 64 Qualitätssicherung 64

Rekonstruktive Beckenchirurgie 133 Renaissance 142 Rotenhausgasse 153

Sarkoidose 98 Soziales Umfeld 199 Spinalanästhesie 173 Staging-Laparotomie 149

Stressharninkontinenz 173 Stressinkontinenz 170, 175 Systemische Applikation 79

Thrombose 6 Tokolyse 215

Transvaginalsonographie 38 Trophoblastpersistenz 29 Tuboovarialabszess 226 Tumorbiologie 133 Tumorresektion 34

Universitätskliniken Wien 164 Urogynäkologie 139

Vaginal-operative Tradition 142 Vaginale Chirurgie 139 Vulvakarzinom T1, NO-1 209

Wachstumsfaktoren 12 Wassergeburt 46

Zervikale epitheliale Neoplasien 188

Zervixkarzinom 16

-, operative Therapie 133

-, Zellinien 85

Zukunftsaspekte 149

244 